\title{
Screening action potentials: the power of light
}

\author{
Lars Kaestner ${ }^{1,2}$ and Peter Lipp ${ }^{1,2}$ \\ 1 Institute for Molecular Cell Biology, School of Medicine, Saarland University, Homburg/Saar, Germany \\ ${ }^{2}$ Research Center for Molecular Imaging and Screening, Saarland University, Homburg/Saar, Germany
}

\section{Edited by:}

Ralf Franz Kettenhofen, Axiogenesis

$A G$, Germany

Reviewed by:

Erik Renström, Lund University,

Sweden

William A. Coetzee, NYU School of

Medicine, USA

${ }^{*}$ Correspondence:

Lars Kaestner, Institute for Molecular Cell Biology, School of Medicine,

Building 61, 66421 Homburg/Saar,

Germany.

e-mail: lars_kaestner@me.com
Action potentials reflect the concerted activity of all electrogenic constituents in the plasma membrane during the excitation of a cell. Therefore, the action potential is an integrated read out and a promising parameter to detect electrophysiological failures or modifications thereof in diagnosis as well as in drug screens. Cellular action potentials can be recorded by optical approaches. To fulfill the pre-requirements to scale up for, e.g., pharmacological screens the following preparatory work has to be provided: (i) model cells under investigation need to represent target cells in the best possible manner; (ii) optical sensors that can be either small molecule dyes or genetically encoded potential probes need to provide a reliable read out with minimal interaction with the naive behavior of the cells and (iii) devices need to be capable to stimulate the cells, read out the signals with the appropriate speed as well as provide the capacity for a sufficient throughput. Here we discuss several scenarios for all three categories in the field of cardiac physiology and pharmacology and provide a perspective to use the power of light in screening cardiac action potentials.

Keywords: cardiac action potentials, membrane potential dyes, genetically encoded membrane potential sensors, human cardiac myocytes, optical screens

\section{INTRODUCTION}

Pharmacological screening of ion channels is traditionally performed by the patch-clamp technique, related tools, and their automated incarnations or indirect fluorescence as well as luminescence based end-point measurements. There are good reasons why the patch-clamp technique is the gold standard in investigating ion channels, such as the possibility to isolate specific currents or to characterize single channels. However, there are scientific questions, pharmacological approaches, or medical requirements, when the concert of electrogenic membrane transport entities are of particular interest. Such a situation is, e.g., the measurement of action potentials for cardiac safety issues. The action potential duration can be regarded as a cellular equivalent of the QT-Interval in electrocardiograms (Arrigoni and Crivori, 2007). Since the investigation of action potential considers all electrogenic elements and is thus not limited to a single entity, e.g., hERG channels, the screening of action potentials bears a conceptional advantage. Action potentials can be measured optically for almost four decades (Salzberg et al., 1973). Beside the measurement of action potentials in isolated primary cells, optical methods can follow cellular action potentials and their interplay in tissue slices and entire organs (e.g., Jin et al., 2002; Lee et al., 2011). Here we describe recent advances and promising perspectives in screening cardiac action potentials.

\section{CELLULAR SYSTEMS}

When it comes to cellular systems for cardiac safety screens we find an inherent conflict; on one hand cells should be as close as possible to human situation and on the other hand simplicity of operation. Since optical approaches operate contact free, they bear the potential to screen even very fragile cells like human atrial myocytes as depicted in Figure 1A. Human cardiomyocytes, either atrial myocytes from appendages after surgery involving heart-lung machines (Kaestner and Lipp, 2007) or ventricular myocytes taken from biopsies, facilitate screening drugs for personalized medication. However, for pharmacological safety screens one would need to switch to animal models: for adult ventricular myocytes we recently described a reliable isolation procedure (Kaestner et al., 2009) as well as optimized culture conditions (Viero et al., 2008; Hammer et al., 2010). Furthermore we compared neonatal and adult cardiomyocytes in a basic pharmacological profiling (Tian et al., 2011), showing that neonatal cells do not reflect the adult situation at all. The present guidelines of FDA and EMEA for cardiac safety screens (Food and Drug Administration, HHS, 2005) explicitly exclude rodents for such investigations because adult animals almost completely lack hERG expression. However, neonatal cardiomyocytes express hERG (Kaestner et al., 2011a; Tian et al., 2011) and therefore the guidelines may need a revision. Furthermore, it might have to be considered that during aging and many cardiac diseases re-expression of fetal and/or neonatal genes might change this situation. Very promising are the ongoing developments and refinements to differentiate cardiac myocytes from embryonic or pluripotent stem cells (e.g., Kettenhofen and Bohlen, 2008) and (Moretti et al., 2010), respectively. The years to come will show to what extend stem cell derived cardiomyocytes will enter cardiac safety screens - the potential is enormous.

\section{OPTICAL SENSORS}

Since action potentials in cardiomyocytes are convolved by cellular contraction artifacts optical read out need to be performed in a quantitative manner, e.g., based on ratiometric or fluorescence 




c

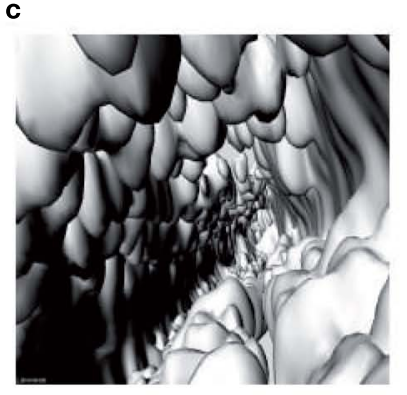

C a

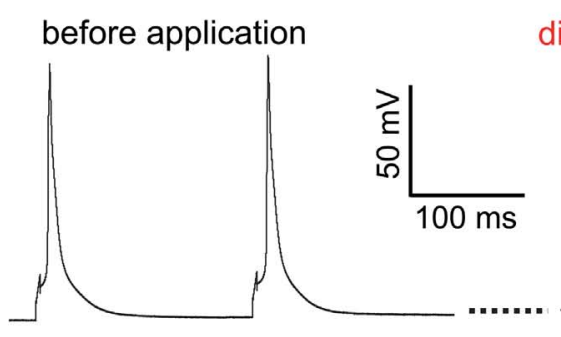

b

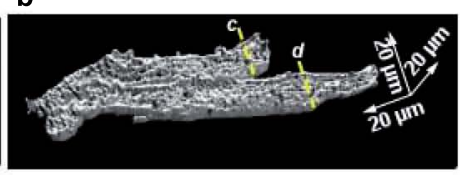

d

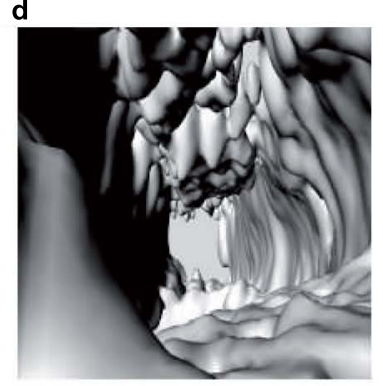

3 min after end of di-8-ANEPPS application
B

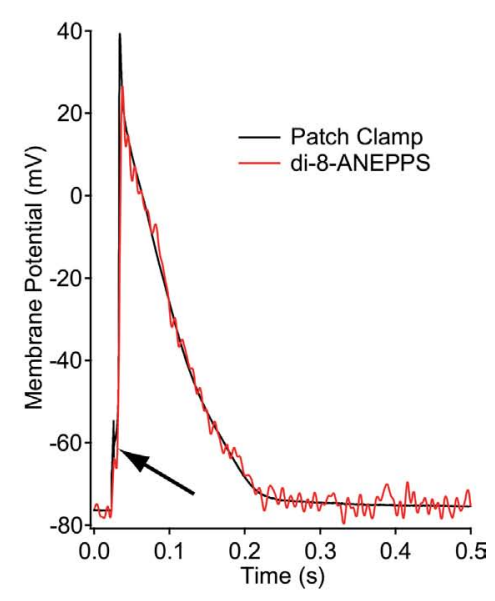

b

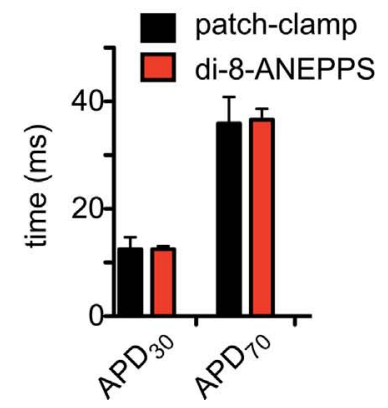

FIGURE 1 | (A) Depicts isolated human atrial myocytes loaded with the membrane potential dye di-8-ANEPPS. Image (a) displays a max. intensity projection of a confocal z-stack of two atrial myocytes, while image (b) depicts a cut open 3D-reconstruction of the same cells. The cells are virtually cut open along the lines $c, d$ in image (b) and the view into the cells from that position is displayed in images (c,d), respectively. (B) Illustrates that the patch-clamp technique (black line) and the optical sensor (red line) measure exactly the same potential. Even the stimulation artifact of the patch-clamp technique to induce an action potential translates into the optical signal (black arrow). (C) Shows that the di-8-ANEPPS, when applied at a concentration of $5 \mu \mathrm{M}$ for $7 \mathrm{~min}$, does not disturb the naive action potential. (a) Displays action potentials recorded by patch-clamp before and after the di-8-ANEPPS staining procedure. (b) Depicts a statistical analysis of action potential duration in cells measured by the patch-clamp technique $(n=94)$ and measured by the optical di-8-ANEPPS read out $(n=214)$. (B,C) are reproduced from Tian et al. (2011) with friendly permission of S. Karger AG (Basel, Switzerland). lifetime approaches. Alternatively, contractions can be suppressed, but this is problematic since compounds such as BDM or cytochalasin $\mathrm{D}$ prolong the action potential in the required concentrations (discussed in Tian et al., 2011). Blebbistatin is a more promising drug, but is easily photoconverted (Tian et al., 2011). For quantitative read-out fluorescence lifetime imaging is inherently too slow (Kaestner et al., 2011b). Therefore we regard ratiometric approaches as the method of choice. Small molecule dyes such as di-4-ANEPPS or di-8-ANEPPS can be used in an excitation or emission ratiometric manner. Although, with the availability of diode based light sources switching times between excitation wavelengths can be fairly short (Lee et al., 2011), emission ratiometric scenarios generate both channels simultaneously allowing at least two times the acquisition speed. For di-8-ANEPPS it was shown that (a) it resembles action potentials from current clamp recordings (e.g., Figure 1B) and (b) the naive action potential is not influenced by the probe, if applied appropriately (Figure 1C; Tian et al., 2011). A promising development is a novel dye, called di-4-ANBDQPQ (Matiukas et al., 2007; Lee et al., 2011), that shows bathochromely shifted spectra and therefore may be used in the presence of GFP, that is often expressed, e.g., as cardiac marker in stem cell environments.

Yet another approach to potential sensitive sensors are genetically encoded potential probes. We published a recent review with particular emphasize on their application in cardiac myocytes (Kaestner et al., 2011b). One of the most promising candidates, the FRET based "Mermaid," is introduced in Figure 2. The use of novel fluorescent proteins (Figure 2A) requires unusual detection channels when compared to the "classical" FRET pair CFP/YFP (Figure 2B). Especially since the mKusabira orange $\mathrm{K}_{\mathrm{K}}$ is prawn to rapid bleaching it is not easy to get the best out of Mermaid (Atsushi Miyawaki and Hidekatzu Tsutsui, personal communication). Since there have been conflicting reports about the amino acid sequence of the linker in Mermaid (Tsutsui et al., 2008; Mutoh et al., 2009), we provide the sequence of the construct (Figure 2C) that was used in our previous studies (Kaestner et al., 2011a; Tian et al., 2011) as well as in Figure 2D. However, genetically 
A



B

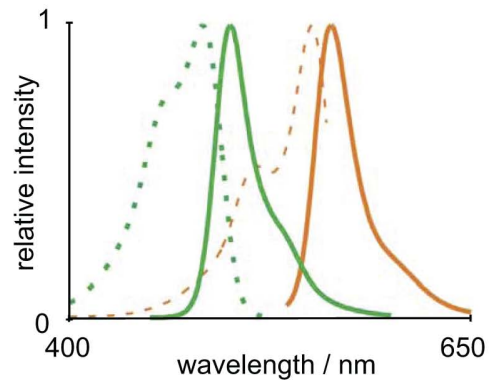

D a

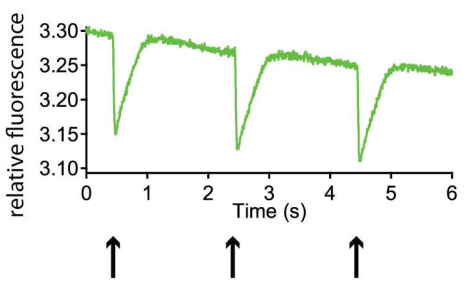

b

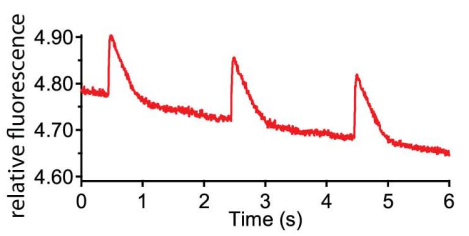

c

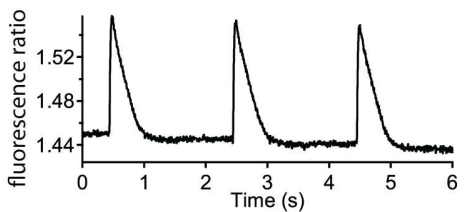

C

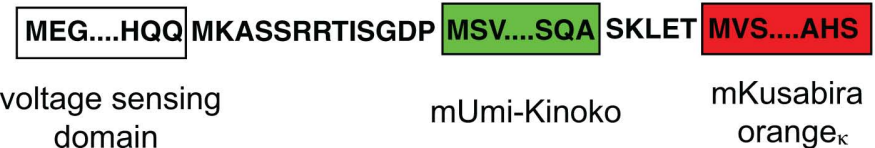

FIGURE 2 | (A) Is a schematic illustration of the FRET based sensor Mermaid containing two coral derived fluorescence proteins, wheras the voltage sensor containing a phosphatase of the sea squirt Ciona intestinalis, provides the base for the conformational change. The spectral properties of these fluorescent proteins are given in (B) (mUmi-Kinoko green lines and mKuabira orange $\mathrm{K}_{\mathrm{K}}$ orange lines; excitation spectra dashed lines and fluorescence spectra solid lines). (A,B) are reprinted by permission from Macmillan Publishers Ltd: Nature Methods (Tsutsui et al., 2008), copyright (2008). Since there have been conflicting reports concerning the linker sequences of Mermaid (Tsutsui et al., 2008; Mutoh et al., 2009), in (C) we provide the linker sequence of the construct used for our investigations [cp. (D)]. (D) Depicts action potential recordings from viral transduced Mermaid in isolated ventricular myocytes. (a,b) Are traces of the fluorescence intensity of the two spectral channels, while (Dc) presents the ratio of (a,b). (D) Is a reprint from Tian et al. (2011) with friendly permission of S. Karger AG (Basel, Switzerland). encoded potential sensors bear a potential for further improvements, especially with respect to their kinetic properties (Kaestner et al., 2011b).

\section{DEVICES}

To perform optical screens there are several requirements for the hardware, comprising three major components: (i) cell substrate and cavity that meet the conditions for optical screens, (ii) a microscope for imaging and (iii) a robotic system that handles the workflow:

(i) A suitable substrate with oleophobic properties compatible with oil immersion objectives on the outside and elastic coating to mimic extracellular matrix on the inside was introduced recently (Muller et al., 2010). This was implemented in 24-well plates, as shown in Figure 3A, but would need further integration into 96 well plates and beyond. For electrical field stimulation of the cells this particular model used carbon electrodes incorporated into the lid. This method has the disadvantage of producing unwanted electrolytic by-products that need to be taken care of, e.g., by more frequent change of the medium. Alternative concepts include cell stimulation by electromagnetic fields with an appropriate coil. Since such a coil would need proximity to the cells there are spatial conflicts with the objective. Another promising alternative to excite cardiomyocytes is the optogenetic manipulation by the expression of channelrhodopsin, a light-activated cation channel, that can induce action potentials (Bruegmann et al., 2010).

(ii) As mentioned above fast read out of membrane potentials requires emission ratiometric approaches that in the past were only performed with photometric techniques (e.g., Hardy et al., 2009; Kaestner et al., 2011a,b). To increase the throughput many cells need to be measured simultaneously. Such properties are offered by systems employing array detectors. Figure 3B depicts a design of a microscope with an integrated image splitter projecting two spectral channels on one camera chip. Recent developments of CMOS camera chips (Coates et al., 2009; Figure 3Ca) provide a technology unifying high acquisition speed (Figure $3 \mathbf{B b}$ ) and good quantum efficiency (Figure 3Bc). Within the near future the latter parameter will improve even further since back thinned scientific CMOS chips will become available. 

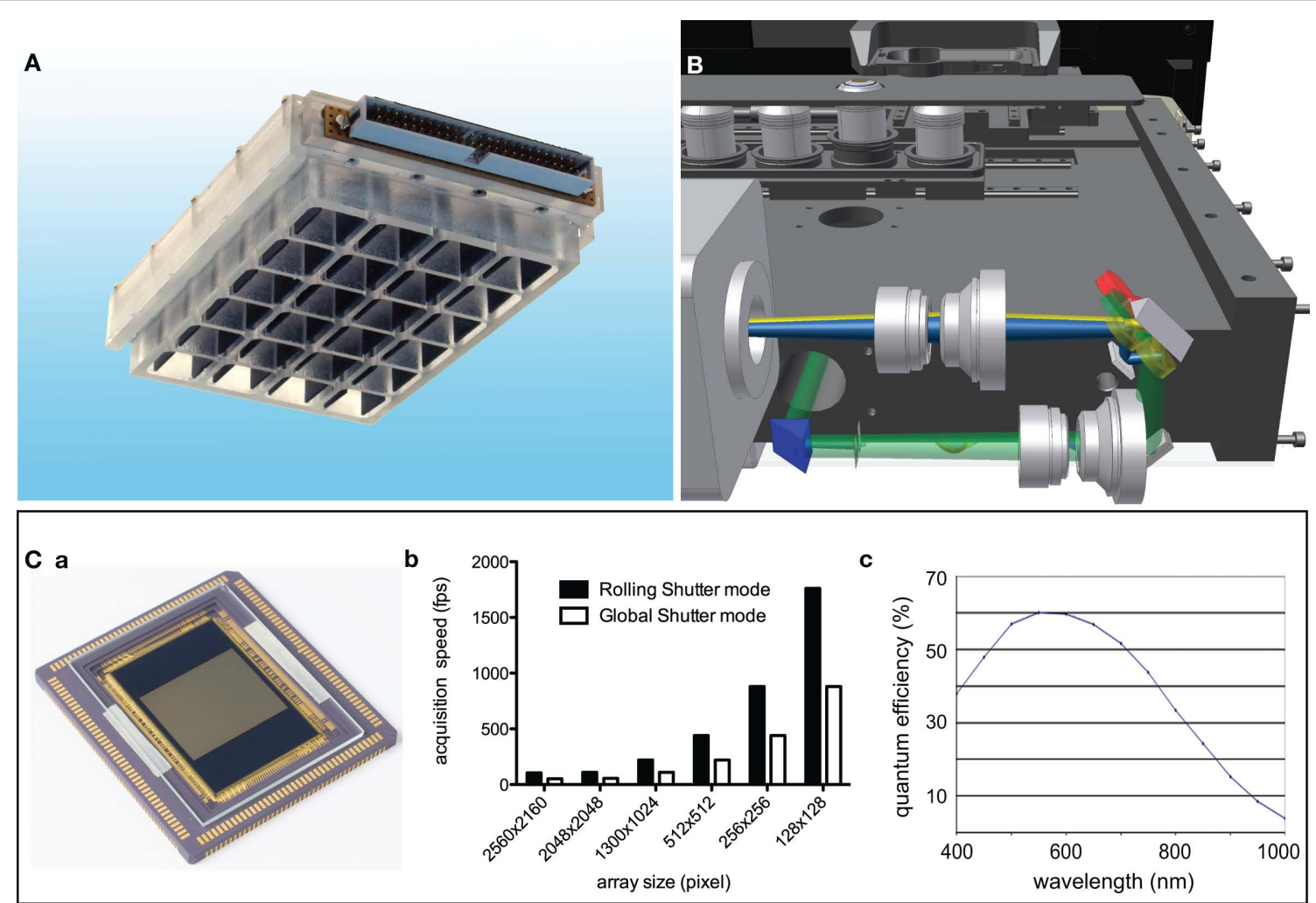

FIGURE 3 | Hardware enabling optical action potential screens. (A) Depicts a 24-well plate allowing an electrical field stimulation of cardiac myocytes under the application of high numerical aperture oil immersion objectives. For a detailed description refer to Muller et al. (2010)). (B) Shows a design of a prototype microscope, which is representative for a new generation of microscopes allowing a precise screen of standard multi-well plates and be able to detect two emission channels simultaneously on array detectors like CCD or CMOS based cameras. (B) Is a courtesy from Prof. Rainer Uhl (TILL I.D. GmbH, Gräfelfing, Germany). (C) Shows the state of the art scientific CMOS sensor (a) and key specifications of that sensor, what are frame rates in the range of $1 \mathrm{kHz}$ (b) and a quantum efficiency of $60 \%$ around $550 \mathrm{~nm}$ (c). (D) Is modified from Coates et al. (2009). (iii) To perform screens an automated plate and liquid handling, electrical stimulation (see above), and a complex microscopy protocol are compulsory. Although each of the elements is readily available on its own, a device combining the necessary hardware and an advanced software allowing online analysis and feedback into the acquisition procedure remains to be developed.

\section{CONCLUSION}

Beside the conceptional advantages of screening action potentials as outlined in the introduction, optical screens have a minimal requirement for the cellular system, since cells do not need to be mechanically manipulated. Therefore it is a very flexible screening approach and eventually the only one that can be applied to intact adult cardiomyocytes, in addition to model cells and stem cell derived cardiomyocytes. Action

\section{REFERENCES}

Arrigoni, C., and Crivori, P. (2007). Assessment of QT liabilities in drug development. Cell Biol. Toxicol. 23, $1-13$.

Bruegmann, T., Malan, D., Hesse, M., Beiert, T., Fuegemann, C. J.,
Fleischmann, B. K., and Sasse, P. (2010). Optogenetic control of heart muscle in vitro and in vivo. Nat. Methods 7, 897-900.

Coates, C., Fowler, B., and Holst, G. (2009). Scientific CMOS Technology - A High-Performance Imaging

potential screening can be combined with other optical measurements, such as those employing GFP for identifying stem cell derived cardiomyocytes (see above) or for imaging calcium (Lee et al., 2011). Additionally, optical screening that takes advantage of genetically encoded potential sensors allows experimental regimes in which cells can be revisited, even after a couple of days. Taken together, the possibilities of optical action potential screening may enable novel fields of application beyond cardiac safety.

\section{ACKNOWLEDGMENTS}

We like to thank Dr. Sandra Ruppenthal for assistance with the sequencing of Mermaid and Mrs. Sarah Schwarz for isolating the human atrial myocytes. This work was supported by the German Federal Ministry for Education and Research (BMBF) and the German Research Foundation (KFO 196).

Breakthrough. Belfast: Andor Technology PLC, Fairchild Imaging, PCO AG, 1-14.

Food and Drug Administration, HHS. (2005). ICH: S7B nonclinical evaluation of the potential for delayed ventricular repolarization (QT interval prolongation) by human pharmaceuticals, Fed. Regist. 70, 61133-61134.

Hammer, K., Ruppenthal, S., Viero, C., Scholz, A., Edelmann, L., Kaestner, L., and Lipp, P. (2010). Remodelling of $\mathrm{Ca} 2+$ handling organelles in adult 
rat ventricular myocytes during long term culture. J. Mol. Cell. Cardiol. 49, 427-437.

Hardy, M. E. L., Pollard, C. E., Small, B. G., Bridgland-Taylor, M., Woods, A. J., Valentin, J., and Abi-Gerges, N. (2009). Validation of a voltagesensitive dye (di-4-ANEPPS)-based method for assessing drug-induced delayed repolarisation in Beagle dog left ventricular midmyocardial myocytes. J. Pharmacol. Toxicol. Methods 60, 94-106.

Jin, W., Zhang, R.-J., and Wu, J.-Y. (2002). Voltage-sensitive dye imaging of population neuronal activity in cortical tissue. J. Neurosci. Methods 115, 13-27.

Kaestner, L., and Lipp, P. (2007). "Nonlinear and ultra high-speed imaging for explorations of the murine and human heart," in Optics in Life Science, Vol. 6633, eds J. Popp and G. von Bally (Munich: SPIE), 66330K1-66330K-10.

Kaestner, L., Scholz, A., Hammer, K., Vecerdea, A., Ruppenthal, S., and Lipp, P. (2009). Isolation and genetic manipulation of adult cardiac myocytes for confocal imaging. J. Vis. Exp. 31. doi: 10.3791/1433

Kaestner, L., Tian, Q., and Lipp, P. (2011a). "Cardiac safety screens: molecular, cellular and optical advancements," in Biomedical Optics
III, Vol. 8089, eds C. P. Lin and V. Ntziachistos (Munich: SPIE), 80890H-1-80890H-6.

Kaestner, L., Tian, Q., and Lipp, P. (2011b). "Action potentials in heart cells," in Fluorescent Proteins II Application of Fluorescent Protein Technology, ed. G. Jung (Heidelberg: Springer), doi: 10.1007/4243_2011_ 28. [Epub ahead of print].

Kettenhofen, R., and Bohlen, H. (2008). Preclinical assessment of cardiac toxicity. Drug Discov. Today 13, 702-707.

Lee, P., Bollensdorff, C., Quinn, T. A., Wuskell, J. P., Loew, L. M., and Kohl, P. (2011). Single-sensor system for spatially-resolved, continuous and multi-parametric optical mapping of cardiac tissue. Heart Rhythm. doi: 10.1016/j.hrthm.2011.03.061. [Epub ahead of print].

Matiukas, A., Mitrea, B. G., Qin, M., Pertsov, A. M., Shvedko, A. G., Warren, M. D., Zaitsev, A. V., Wuskell, J. P., Wei, M. D., Watras, J., and Loew, L. M. (2007). Near-infrared voltage-sensitive fluorescent dyes optimized for optical mapping in blood-perfused myocardium. Heart Rhythm 4, 1441-1451.

Moretti, A., Bellin, M., Welling, A., Jung, C. B., Lam, J. T., BottFlügel, L., Dorn, T., Goedel, A., Höhnke, C., Hofmann, F., Seyfarth,
M., Sinnecker, D., Schömig, A., and Laugwitz, K. L. (2010). Patientspecific induced pluripotent stemcell models for long-QT syndrome. N. Engl. J. Med. 363, 1397-1409.

Muller, O., Tian, Q., Zantl, R., Kahl, V. Lipp, P., and Kaestner, L. (2010). A system for optical high resolution screening of electrical excitable cells. Cell Calcium 47, 224-233.

Mutoh, H., Perron, A., Dimitrov, D. Iwamoto, Y., Akemann, W., Chudakov, D. M., and Knöpfel, T. (2009). Spectrally-resolved response properties of the three most advanced FRET based fluorescent protein voltage probes. PLoS ONE 4, e4555. doi: 10.1371/journal.pone.0004555

Salzberg, B. M., Davila, H. V., and Cohen, L. B. (1973). Optical recording of impulses in individual neurones of an invertebrate central nervous system. Nature 246, 508-509.

Tian, Q., Oberhofer, M., Ruppenthal, S., Scholz, A., Buschmann, V., Tsutsui, H., Miyawaki, A., Zeug, A., Lipp, P., and Kaestner, L. (2011). Optical action potential screening on adult ventricular myocytes as an alternative QT-screen. Cell. Physiol. Biochem. 27, 281-290.

Tsutsui, H., Karasawa, S., Okamura, Y. and Miyawaki, A. (2008). Improving membrane voltage measurements using FRET with new fluorescent proteins. Nat. Methods 5, 683-685.

Viero, C., Kraushaar, U., Ruppenthal, S., Kaestner, L., and Lipp, P. (2008). A primary culture system for sustained expression of a calcium sensor in preserved adult rat ventricular myocytes. Cell Calcium 43, 59-71.

Conflict of Interest Statement: The authors declare that the research was conducted in the absence of any commercial or financial relationships that could be construed as a potential conflict of interest.

Received: 15 June 2011; paper pending published: 27 June 2011; accepted: 12 July 2011; published online: 28 July 2011.

Citation: Kaestner L and Lipp P (2011) Screening action potentials: the power of light. Front. Pharmacol. 2:42. doi: 10.3389/fphar.2011.00042

This article was submitted to Frontiers in Ion Channels and Channelopathies, a specialty of Frontiers in Pharmacology. Copyright (c) 2011 Kaestner and Lipp. This is an open-access article subject to a non-exclusive license between the authors and Frontiers Media SA, which permits use, distribution and reproduction in other forums, provided the original authors and source are credited and other Frontiers conditions are complied with. 